\title{
Object Reminder and Safety Alarm
}

Chi-yau Lin, Chia-nan Ke, Shao-you Cheng, Jane Yung-jen Hsu, and Hao-hua Chu

\author{
Computer Science and Information Engineering, \\ National Taiwan University, Taiwan \\ \{r93922129, r93922109, r93922070, yjhsu, hchu\}@ntu.edu.tw
}

\begin{abstract}
This paper introduces a novel approach to enhancing safety through RFID technology, location tracking, and monitoring person-object interaction. We design and develop RFID-based wearable devices for (1)tracking people's locations, (2)monitoring person-object interactions, and (3)tracking objects' locations. An intelligent object reminder and safety alert system is proposed to relief the common safety-related worries many of us face in our everyday lives - "Where did I leave my keys?", "Did I turn off the stove?", or "Did I close all the windows in my house?" etc. Experimental results on the precision of object identification and location tracking are also presented.
\end{abstract}

\section{Introduction}

This research aims to design an intelligent alert system by tracking the behavior of users living within the space. By attaching cheap, readily available, passive RFID tags on everyday objects, such as wallets, key chains, briefcases, shoes, spoons, trash cans, refrigerators, paintings, floors and doors, etc. and embedding tiny, mobile RFID readers on wearable personal items such as rings or a watches, the smart environment can unobtrusively monitor human interactions with these RFID-tagged objects in the physical space. By monitoring such person-object interactions and tracking people's indoor locations, we can infer high-level safety-related activity context, such as a person who turns on a stove in the kitchen (touching the stove knob) and then walks away for a long time (he/she may forget to turn off the stove), a person who opens a window and then leaves home (he or she may be in a risk of burglary), a left-along toddler who holds small items (such as coins, buttons, marbles, beads) and is in high risk of choking on them, etc.

In Section 2, we start by describing the hardware devices and infrastructure. Section 3 presents the proposed multiagent system architecture. Experimental results are given in Section 4 followed by related work and conclusion in Sections 5 and 6 .

\section{Hardware Devices}

In the proposed system, we attach short-range RFID tags to everyday objects for identification. Meanwhile, RFID readers are embedded in wearable personal items, such as finger rings or wrist watches, to identify tagged objects that are being handled by the user.

(C) IFIP International Federation for Information Processing 2005 
For location tracking, a user carries a PDA equipped with the Ekahau Positioning Engine 2.1 (EPE). It utilizes existing Wi-Fi network infrastructure to to facilitate user mobility and asset visibility.

\section{System Architecture}

Two kinds of sensed data are used in our system. As Figure 1 depicts, one sort is RFIDtagged object and the other one is Wi-Fi signal via WLAN network. Our system is a multi-agent based system equipped with wearable RFID reader and Ekahau Wi-Fi software positioning engine. Besides, three agents are built to manage sensed data and cooperate in our system.

The first is Object Tracker Agent. From our wearable RFID-Tag reader, it can read the tag id embedded on the surface of objects. Furthermore, it can identify the object from its tag id, and other properties such as category, owner and RFID information that the object has from its object domain mapping.

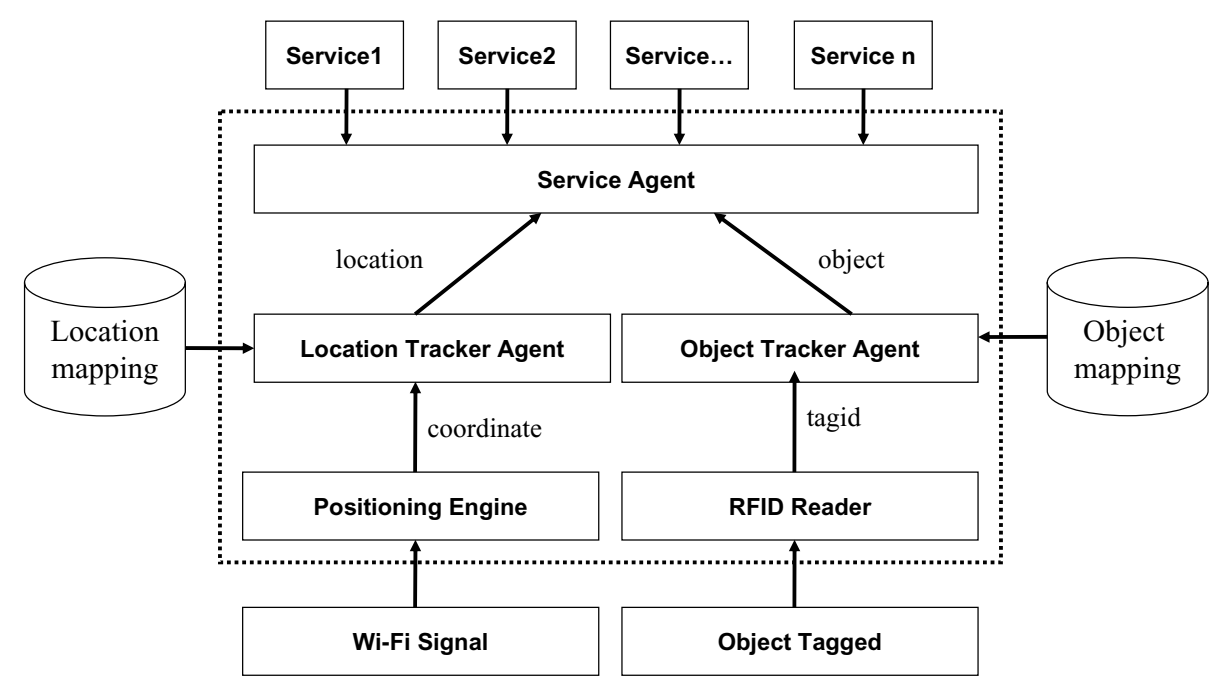

Fig. 1. System architecture based on multi-agents system

The Location Tracker Agent can receive the data transmitted from Ekahau Wi-Fi positioning engine. The Ekahau positioning system returns the coordinates of people's location. The Location Tracker Agent translates the coordinates into places that people can understand.

The last agent is Service Agent. It can supply services to people. The Service Agent can communicate with Object Tracker Agent and Location Tracker Agent, while people want certain service. The details are presented in section 3.3.

Finally, we will describe how the agents communicate, pass message, and parse message to handle the request. 


\subsection{Object Tracker Agent}

Our Object Tracker Agent is a reactive agent. Based on the RFID system, it can percept the object when human touched.

Object Recognition. The Object Tracker Agent can recognize the object that the person held. And it is able to process the data in logic, because it can use rules to make inference, choose courses of action and answer questions. What is the knowledge base in the Object Tracker Agent? We built an object domain with an explicit description of an object. Besides, Object Tracker Agent not only maintains the data of objects, it can also communicate with other agents through a interaction protocol.

\subsection{Location Tracker Agent}

In EPE 2.1, the location means a coordinate in a plat map with a layer of certain building. But for our Location Tracker Agent, it can recognize the physical location of the person, instead of just a coordinate.

Location Tracking with Mobile Device. Location Tracker Agent is built in our notebook which has installed EPE 2.1. And Ekahau Client is installed in the notebook and PDA. EPE 2.1 will monitor the location of Ekahau Clients. If the location changing, Location Tracker Agent captures the current time and stores the location information in its repository. It translates the location information into Area instead of the coordinate return from EPE 2.1. Area means a block like lab room, toilet, hallway, and so on. Figure 2 shows the Area we defined in our experimental environment.

\subsection{Service Agent}

The Service Agent can supply various services while people request. First, we implemented the simple combination of Location Tracker Agent and Object Tracker Agent to find the location of an object. Last, we set rules to detect if any dangerous object ever touched by human, and monitor the interaction between human and objects.

Object Locating. Figure 3 describes a simple scenario for our Object Locating Service. The situation occurs to everyone in our daily life. Searching a certain thing is really tedious for people, especially when we need the item in emergency. So how to find the item as soon as possible is a really practical problem. We solved the problem with combination of object and location information.

Safety Alarm. In the same scenario, we describe another scenario in Figure 4 to provide our Safety Alarm Service. The service can remind John to avoid an accident due to his carelessness.

Service Features. Our services focus on some practical problems and have many features.

First, our smart device is a wearable item that embedded a RFID reader, and a personal server in his pocket. Second, our system is no range limit. Second, our smart 


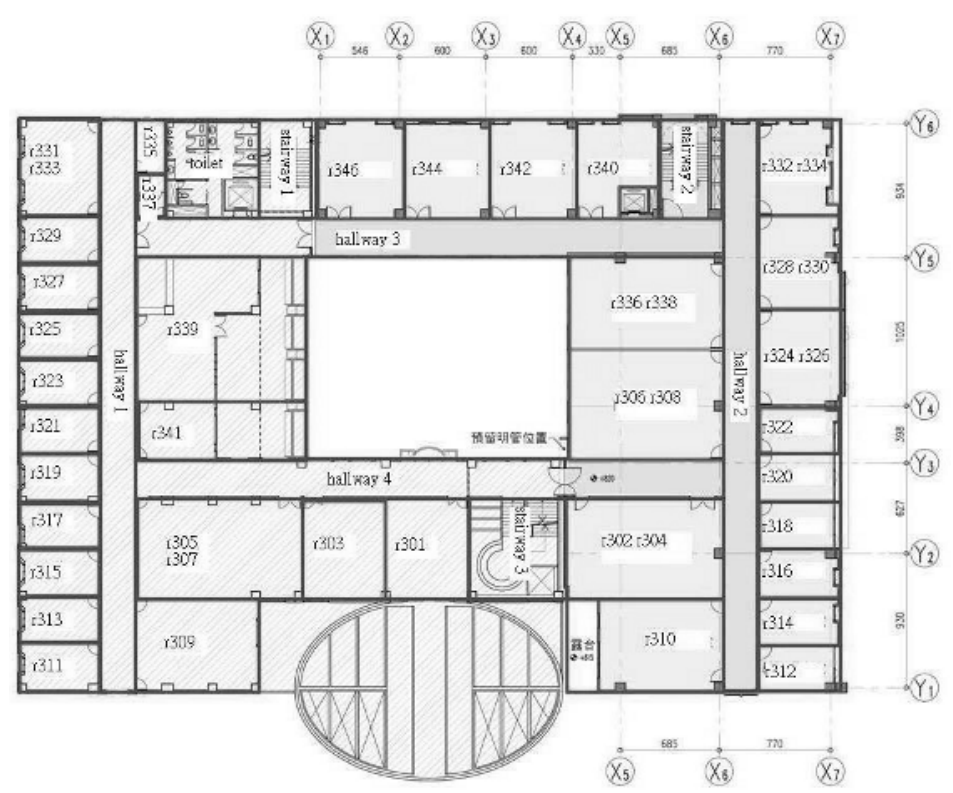

Fig. 2. The 3rd floor plan of Computer Science and Information Engineering building at National Taiwan University

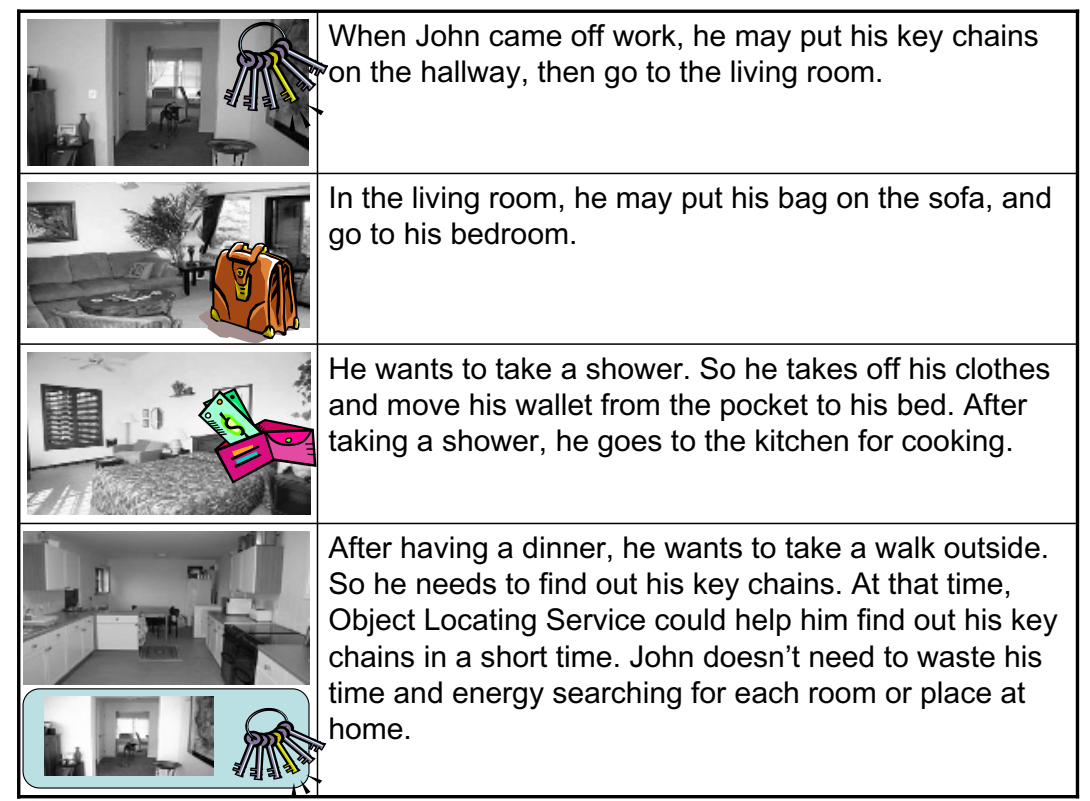

Fig. 3. Object Locating Scenario 


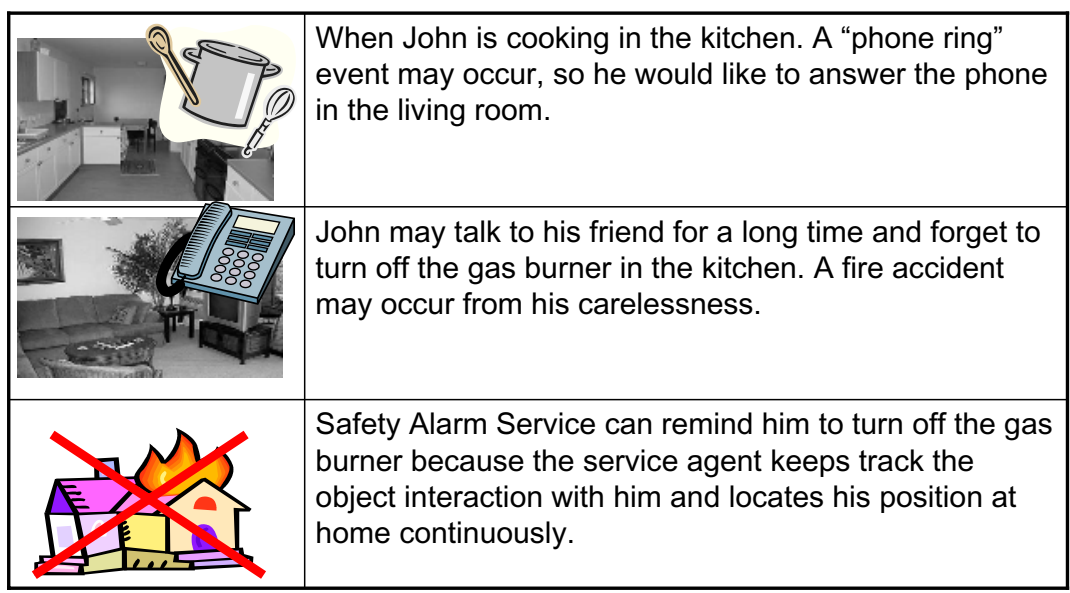

Fig. 4. Safety Alarm Scenario

device is easy to use, because our smart device is an agent-based system. We design and implement a multiagent system, so our system can update automatically. In other words, our system is less demand for user intervention because of the autonomous of agents, the person will have a clear user interface, and needn't to set any preference in advance.

\subsection{Agent Communication}

The communication of our agents is a FIPA like contract interaction protocol specification. Our communication message format contains the identification of which agent sends a request, and which action that the agent requests another agent to do, and the time when an agent makes a request, and other information needed to pass to another agent. When the agent received a request, it can understand the meaning of the request,

Table 1. Communication Messages

Messages for Object Locating

\begin{tabular}{|l|l|c|l|l|}
\hline \multicolumn{1}{|c|}{ Sender } & \multicolumn{1}{c|}{ Receiver } & Request & Time Stamp & \multicolumn{1}{c|}{ Content } \\
\hline Service Agent & Object Tracker Agent & request object & system time & object name \\
\hline Object Tracker Agent & Service Agent & - & system time & time or location \\
\hline Service Agent & Object Tracker Agent & get location & system time & object name \\
\hline Location Tracker Agent & Service Agent & - & system time & object location \\
\hline
\end{tabular}

Messages for Safety Alarm

\begin{tabular}{|l|l|c|l|c|}
\hline \multicolumn{1}{|c|}{ Sender } & \multicolumn{1}{c|}{ Receiver } & Request & Time Stamp & Content \\
\hline Service Agent & Object Tracker Agent & dangerous object & system time & - \\
\hline Object Tracker Agent & Search Agent & - & system time & Yes or No \\
\hline Service Agent & Location Tracker Agent & location changed & system time & - \\
\hline Location Tracker Agent & Service Agent & - & system time & Yes or No \\
\hline
\end{tabular}


and response to the request, therefore the agents can negotiate with each other. Table 1 is the message format that we defined for agents.

Agents in Object Locating Service. People always forgot the location where they put their object, the service of Object Locating will remind him his missing object. Figure 5(a) shows the detail of interaction among agents in Object Locating Service.

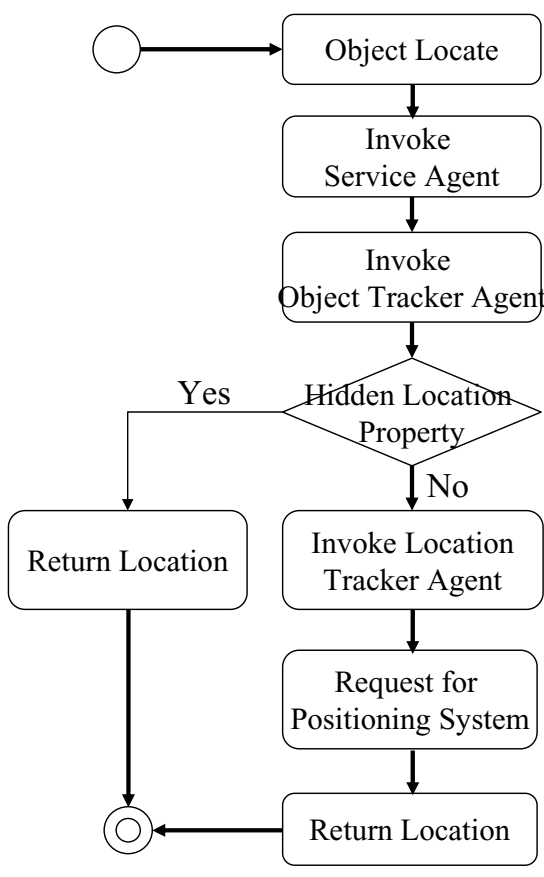

(a) Object Locating



(b) Safety Alarm

Fig. 5. Activity Diagram for Object Locating and Safety Alarm Services

Agents in Safety Alarm Service. People may ask the service of Safety Alarm to guard their safety in home or other places. The Service Agent will invoke Object Tracker Agent to monitor whether the object user touched has any potential danger, such as steam iron or gas burner. Figure 5(b) shows detail of agents' activities for this service.

\section{Experiments}

We conducted our experiments in the 3rd floor of the Computer Science and Information Engineering at National Taiwan University. And we put everyday objects with RFID tag in our laboratory. Besides, we are equipped with wearable item with RFID reader, and use RS232 transmission line to connect our personal server. Figure 6 shows the equipments and objects we touched in the experiment. 

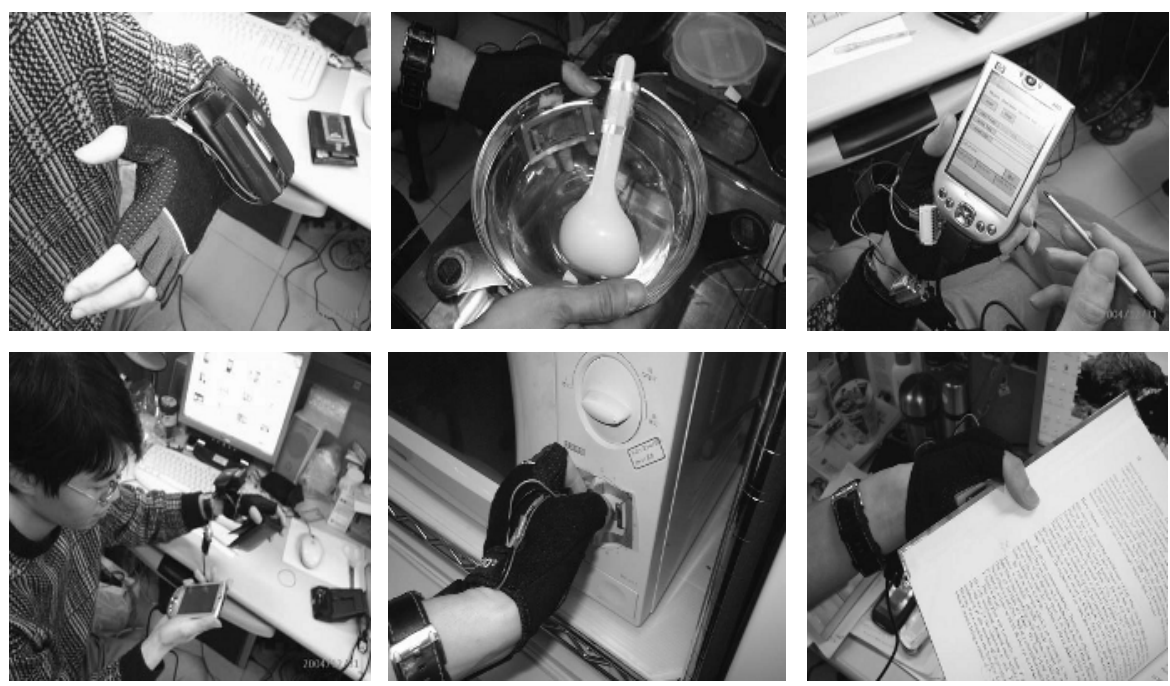

Fig. 6. Equipments and objects we touched in the experiment.

\subsection{Object Recognition}

We tested several objects when we touched the object in a certain condition. First, we attached lots of tags to our everyday objects. Objects include camera, microwave switch, book, wallet, PDA, mobile phone, mouse, pitcher, bowl, and spoon. These objects are placed in our laboratory. We used our wearable item with RFID reader and PDA as our sensing devices. We tested 20 times for each object.

Table 2 shows the experimental results for object identification. If the object is small enough to hold in hand, the precision will be higher, such as PDA, mobile phone and spoon. In order to have better performance, we suggest that the tag must be attached

Table 2. Precision for tagged object reads

\begin{tabular}{llccc}
\hline Object Name & Test Condition & Tag Position & Success Reads Precision (\%) \\
\hline Digital camera & Taking pictures & Left hand side & 18 & 90 \\
Microwave switch Turning on & Switch & 8 & 40 \\
Book & Reading & Cover & 16 & 80 \\
Wallet & Retrieving money & Back & 14 & 70 \\
PDA & Holding & Back & 18 & 90 \\
Mobile phone & Talking & Back & 19 & 90 \\
Mouse & Web browsing & Middle & 14 & 70 \\
Pitcher & Holding & Side & 11 & 55 \\
Bowl & Drinking & Side & 10 & 50 \\
Spoon & Spooning up & Handle & 19 & 95 \\
\hline
\end{tabular}


to the objects according to human's behavior. The performance of object recognition is decided by the human's behavior and the interference of radio frequency.

\subsection{The Problem in Location Tracking}

We tested our Location Tracker Agent in the 3rd floor of the Computer Science Information Engineering building at National Taiwan University. We find several factors that will influence our results.

EPE 2.1 uses Wi-Fi networking technology, and the signal strength is not stable. Numbers of people, objects changed in the environment and the geography of access points will influence the signal strength that the client received. So the estimation of location position may have inaccuracy. If the rectangle of an area is too small, and the EPE 2.1 is unstable, then the location of device will change easier in different area. Therefore the size of our rectangle area is also an important issue in our research domain. Too big would lead user into a confusion about the location, and too small, the change would give user error information. Sometimes, the device doesn't change its location, but the result shows that it changed. Some areas like hallway (shown in Fig 2) have this problem seriously, because hallway is a narrow area, the system may misunderstand it as a room easily.

\section{Related Work}

People already use many kind of assistant tools for managing their daily routines and schedule. Reminder tools like to-do list or post-it note are very common used in our daily life to assist people to remember what they need to do next or bring something they forget. While tasks and plans are getting more and more complex, technology like personal information manager (PIM) or electronic calendar, can help us easily handle complicated and numerous personal objects. When we are now in pervasive computing environment, taking advantages of rich contexts provides such reminder system more features and intelligence. CybreMinder [1] is an early research of using context information for delivering messages via different ways in right situation. Messages can be voice message, e-mail or displaying on nearby displays. The user context here can be a person, location, time, activity, etc. In the similar way, Gate Reminder [2] also tries different reminding ways through different kind of interfaces deployed in a future smart home, as its name, this project sets up a home appliance located in the front door to achieve transparent interaction using RFID technology. The RFID reader will real time capture people and objects identification by passive tags, match home member's item list with their predefined schedule, then provide the effective reminders according to the right contexts. In their work, a series of design principle and user studies were given.

Another kind of application aims to use reminder as the assisted cognition tool for caring the elders and people with memory impairment. Autominder [3] is a project to support activity reminder and help clients remain their daily living activities, such as eating, performing hygiene, taking medicine and toileting. It keeps the client's activity model for further planning and has scalability for addition or modification of an activity. This system can be further implemented on a nurse robot for home care. 
While wearable computer and sensor technology are easily deployed and embedded into system, collecting contexts is thus no more a complicated mission. One of the most potential topic of context inference is to infer human activity. A possible approach is to detect person-object interaction with a wearable device [4]. Recently, many researchers interest on exploiting RFID technology for person and object identification, furthermore, they can track what they have identified. The main concept is to perceive interaction between human and physical world [5]. Schmidt et al [6] suggest a wearable RFID-tag reader to handle tagged objects among explicit human-computer interaction. Pederson [7] has a similar device worn on his finger to track knowledge work actions in the office environment. The difference from his work and former systems is that he considers the location context, but sensing range is restricted in the local office.

The Guide project develops the iGlove [8,9], a glove-based RFID reader with wireless communication, to infer activities of daily living. They successfully infer different types of activity from object-touch sequence. However, they do not combine location information to enhance the inference accuracy. The closest to our approach in the application level is the work developed by Borriello et al [10]. They try to remind people of misplace objects by monitoring them with two long range RFID readers housing in the door way. The personal server will receive item contexts from two readers and check through the personal data stored on it when user crossing through the door. In addition, the location module predicts the possible location destination from many inputs, e.g. calendar, user's schedule and objects sensed. Then, the reminder application generates reminders depending on rules.

\section{Conclusion and Future Work}

An intelligent alert system has been proposed to provide object reminder and safety alarm services. In our sample scenarios, potentially dangerous objects, such as gas burner and iron, are tagged. We have implemented the capability to track when and where any tagged object was touched. For example, a user may go to the kitchen to cook or go to the laundry room to iron his clothes. He may be interrupted by unrelated events like an "incoming telephone call" or a "door bell". The user may be distracted from what he was doing before and forget to turn off the gas burner or to unplug the iron in time. Our system is designed to reduce such safety risks.

We plan to add landmarks in our experimental environment by attaching RFID tags to object that are not easily movable, e.g. heavy furniture or fixtures. When the user touches any landmark, its pre-defined position will substitute for the coarse position obtained from Ekahau. For example, givenn a drinking fountain as a landmark in our lab r335, the Location Tracker Agent will obtain the precise location from the landmark when a user touches the drinking fountain. As a result, we know the user is in r335 for sure. Furthermore, we plan to infer additional activities by tracking interactions among users based on their locations detected by Ekahau.

\section{Acknowledgements}

This work is partially supported by the National Science Council of Taiwan, R.O.C, under grant NSC-93-2218-E-002-148. 


\section{References}

1. Dey, A.K., Abowd, G.D.: Cybreminder: A context-aware system for supporting reminders. In: Proceedings of the 2nd international symposium on Handheld and Ubiquitous Computing (HUC '00), London, UK, Springer-Verlag (2000) 172-186

2. Kim, S.W., Kim, M.C., Park, S.H., Jin, Y.K., Choi, W.S.: Gate reminder: A design case of a smart reminder. In: Proceedings of the 2004 conference on Designing interactive systems (DIS '04), New York, NY, USA, ACM Press (2004) 81-90

3. Pollack, M.E., Brown, L., Colbry, D., McCarthy, C.E., Orosz, C., Peintner, B., Ramakrishnan, S., Tsamardinos, I.: Autominder: An intelligent cognitive orthotic system for people with memory impairment. Robotics and Autonomous Systems 44 (2003) 273-282

4. Fishkin, K., Jiang, B., Philipose, M., Roy, S.: I sense a disturbance in the force: Long-range detection of interactions with RFID-tagged objects. In: Proceedings of Sixth International Conference on Ubiquitous Computing (UbiComp 2004). (2004) 268-282

5. Want, R., Fishkin, K.P., Gujar, A., Harrison, B.L.: Bridging physical and virtual worlds with electronic tags. In: Proceedings of the SIGCHI conference on Human factors in computing systems (CHI '99), New York, NY, USA, ACM Press (1999) 370-377

6. Schmidt, A., Gellersen, H.W., Merz, C.: Enabling implicit human computer interaction - a wearable rfid-tag reader. In: Proceedings of International Symposium on Wearable Computers (ISWC2000). (2000) 193-194

7. Pederson, T.: Magic touch: A simple object location tracking system enabling the development of physical-virtual artefacts in office environments. Personal and Ubiquitous Computing 5 (2001) 54-57

8. Philipose, M., Fishkin, K.P., Fox, D., Kautz, H., Patterson, D., Perkowitz, M.: Guide: Towards understanding daily life via auto-identification and statistical analysis. In: Proceedings of UbiHealth 2003: The 2nd International Workshop on Ubiquitous Computing for Pervasive Healthcare Applications. (2003)

9. Philipose, M., Fishkin, K.P., Perkowitz, M., Patterson, D.J., Fox, D., Kautz, H., Hhnel, D.: Inferring activities from interactions with objects. IEEE Pervasive Computing 3 (2004) 5057

10. Borriello, G., Brunette, W., Hall, M., Hartung, C., Tangney, C.: Reminding about tagged objects using passive rfids. In: Proceedings of Ubiquitous Computing: 6th International Conference (UbiComp 2004), Springer (2004) 36-53 